\title{
The Association between Home Environment and Quality of Life in Children and Adolescents in Hangzhou City, China
}

\author{
Xianhong Huang ${ }^{1} \cdot$ Le Hua $^{2} \cdot$ Xueyang Zhou ${ }^{3} \cdot$ Hao Zhang $^{1} \cdot$ Meng Zhang $^{1} \cdot$ Sheng Wang ${ }^{1} \cdot$ Shangren Qin ${ }^{1} \cdot$ \\ Jie Chen ${ }^{3} \cdot$ XiaoHe Wang ${ }^{1}$
}

Accepted: 26 March 2021 / Published online: 14 April 2021

(c) The Author(s) 2021

\begin{abstract}
To investigate the influence of the home environment, defined as family socioeconomic status (SES) (parent education level, household income), student resource-mediated SES (access to nutritional resources and cognitively stimulating experiences), reading ability, and difficulty with homework on quality of life in children and adolescents residing in urban and suburban areas in Hangzhou City, Zhejiang Province, China. This study included 3200 Grade 3-6 students from 8 elementary schools in Hangzhou City. Assessments included questionnaires that evaluated student quality of life, family SES, resource-mediated SES (dietary behavior and the home literacy environment), reading ability, and difficulty with homework. The effects of the home environment on student quality of life were analyzed by univariate analysis, multiple linear regression analysis, and structural equation modeling. Overall, $80.6 \%$ of students had a medium or better quality of life. Young age (Grade 3 or 4), female sex, household income of 10000-15000 RMB, high breakfast consumption, daily intake of fruit, a balanced diet, and good reading habits were positively correlated with student quality of life $(P<0.05)$, while overuse of electronic devices was negatively correlated with quality of life $(P<0.05)$. Dietary behaviors, home literacy environment, and student reading ability and difficulty with homework directly affected quality of life. Family SES indirectly affected student quality of life. Children and adolescents in China should have access to good nutrition and cognitively stimulating experiences to enhance their well-being and provide them with social and academic advantages.
\end{abstract}

Keywords Quality of life $\cdot$ Home literacy environment $\cdot$ Dietary behavior $\cdot$ Social economic status $\cdot$ Structural equation model

\section{Highlights}

- The influence of the home environment on quality of life in students in China was explored with structural equation modeling.

- Student quality of life was directly affected by diet, the literacy environment, reading ability and difficulty with homework.

- Student quality of life was indirectly affected by family socioeconomic status.

- These findings will inform the development of programs that promote improved quality of life in Chinese children.

Supplementary information The online version contains supplementary material available at https://doi.org/10.1007/s10826021-01951-1.

XiaoHe Wang

xhewang@163.com

1 Department of Health Service Management, School of Medicine Hangzhou Normal University, Hangzhou, China
Childhood and adolescence represent crucial phases in the development of physical, psychological, behavioral, and social maturity (Hosokawa and Katsura, 2017; Lee \& Jackson, 2017; Rashid et al., 2018; Zou et al., 2018). During

2 Affiliated Xixi Hospital, College of Medicine, Zhejiang University, Hangzhou, China

3 The First Affiliated hospital, College of Medicine, Zhejiang University, Hangzhou, China 
these phases, family socioeconomic status (SES) and the home environment influence child and adolescent quality of life (QOL), defined as their physical, emotional, and social well-being (Maatta et al., 2017).

Widely used markers of SES include social indicators, such as occupation and educational level, and economic indicators that are material and resource related (Roubinov \& Boyce, 2017), such as income. The link between SES and QOL in children and adolescents is mediated by accessibility to resources, including nutrition and cognitively stimulating experiences. Nutritional intake influences child and adolescent growth and development, cognitive ability, immunity, morbidity, and mortality, with poor nutrition a key component of poor health (Boe et al., 2018; Bradley \& Corwyn, 2002; Hong, 2007). Access to cognitively stimulating experiences provides children and adolescents direct and peer or adult-mediated learning opportunities that impact their cognitive ability and potential for benefiting from school (Bradley \& Corwyn, 2002; Rowland et al., 2018; Russell et al., 2018). The connection between SES, access to cognitively stimulating experiences, and QOL in children and adolescents is related to parental behavior (Jin $\& \mathrm{Lu}, 2017)$. High-SES parents, defined as those with better education and an economic advantage, typically spend more time reading and communicating with their children and adolescents than low-SES parents (Sun et al., 2013). This might be because higher-SES parents have the time and income to make better interpersonal and material investments in their children's development than lower-SES parents, who must focus on more basic needs (Sohr-Preston et al., 2013). A good home literacy environment is positively related to child and adolescent language and literacy development and might improve child and adolescent reading ability (He et al., 2014; Noble et al., 2006; Sun et al., 2013). Children and adolescents with reading difficulties might experience concomitant psychosocial problems in three dimensions, including self-belief, social cognitive ability, and interpersonal ability (Nathan, 2006). In Hungary, children $<18$ years with a reading disability had a lower QOL than controls without a reading disability (Balazs et al., 2016).

Studies that investigated the influence of the home environment on QOL in children and adolescents focused on children and adolescents with chronic disease or those who were obese. Research that investigated the QOL in children and adolescents in the general population is limited and mainly explored associations between family economic status, parenteral education level, family structure, number of siblings, household crowding, and parenting style (Hosokawa \& Katsura, 2017; Lee \& Jackson, 2017; Ran et al., 2018; Rashid et al., 2018; Zou et al., 2018). In the United States, socioeconomic disadvantages were associated with a significant negative impact on the cognitive achievement of children aged 1-9 years (Lee \& Jackson, 2017). In Japan, family income was related to social skills in preschoolers aged 5 years, and maternal and paternal education levels were related to internalizing and externalizing problems in first graders aged 6 years (Hosokawa \& Katsura, 2017) In Brazil (Paula et al., 2012), clinical, socioeconomic and home environment (family structure; number of siblings; use of cigarettes, alcohol and drugs in the family; household overcrowding) factors exerted a negative impact on the oral health-related QOL of schoolchildren aged 12 years. In Wuhan, China, youth optimism was a mechanism by which family SES was associated with life satisfaction in children and adolescents from primary and high schools (Zou et al., 2018). In Shapingba district, Chongqing, China, inadequate health literacy might have contributed to poor QOL among junior middle school students.

Reports on the influence of other factors on the QOL in children and adolescents in China are limited. Compared with adults, QOL research studies in children and adolescents in China are scarce, possibly because of the relatively stronger emphasis on parent-centeredness than childcenteredness in Chinese culture (Daniel \& Britta, 2007). Previous studies that investigated related topics used traditional statistical methodologies, such as single factor and multiple linear regression analysis (Matthews et al., 2014; Paula et al., 2012; Wang et al., 2007). However, when applying traditional multiple linear regression analysis, the dependent variable and independent variables were defined and only the direct effect between variables was determined. The effect of latent variables, such as factors in the home environment, can only be inferred from other variables that are observed. Structural equation modeling uses a combination of factor and multiple regression analysis that indicates measurement errors, and represents, estimates, and tests a theoretical network between variables (Joreskog \& Sorbom, 1979; Meuleners et al., 2003). Therefore, structural equation modeling evaluates the relationship between latent constructs and observed variables. This study investigated the QOL in children and adolescents residing in urban and suburban areas in Hangzhou City, Zhejiang Province, China using structural equation modeling. Hangzhou City is an economically developed municipality that is located on the southeast coast of China with a per capita GDP of USD 20,419 2017. This study explored how the home environment, defined as family SES (parent education level, household income), resource-mediated SES (access to nutritional resources and cognitively stimulating experiences), reading ability, and difficulty with homework directly and indirectly influenced the QOL in children and adolescents in Hangzhou City. SES, reading ability and difficulty with homework were chosen as the variables measured in this study because evidence suggests (Bradley \& Corwyn, 2002) that the effect of SES on child health is 
mediated by the availability of environmental (reading and nutritional) resources and psychological factors, which influence reading ability and difficulty with homework. Cognitive stimulation is a particularly important consideration. Low-SES children lack resources and experience, which limits the development of their cognitive abilities, reflected by their reading skills, and their potential to benefit from school. In addition, exposure to resources and culture is a mediating variable between SES and child intelligence or academic performance and behavioral problems (Poulain et al., 2020). Understanding how inequalities in the home environment effect children and adolescents informs the development of programs that promote improved QOL in Chinese children.

\section{Methods}

\section{Participants}

The sample include 3200 students with a mean age of 11.19 years (standard deviation $[\mathrm{SD}]=1.96$, range 9-14 years). Based on a dichotomous (male/female) measure of gender, $53.8 \%$ of the students were male and $46.2 \%$ of the students were female. There were 848 (26.5\%) Grade 6 students, 768 (24.0\%) Grade 5 students, 836 (26.1\%) Grade 4 students, and 748 (23.4\%) Grade 3 students. Most of the students (96.9\%) lived with both biological parents, who were married. In addition, this study included 3200 parents (89.3\% mothers) that reported on their children. Parents had a mean age of 38.67 years $(\mathrm{SD}=4.21) .52 .6 \%$ of parents lived in urban areas, and $47.4 \%$ of parents lived in the suburbs. For economic status, 1075 (33.6\%), 1217 (38.0\%), and 908 (28.4\%) families had an income CNY <10000, CNY 10,000-15,000, or $\mathrm{CNY}>15,000$ per month, respectively. Regarding parents occupation, $29.6 \%$ of fathers were professional technical staff, and $22.9 \%$ of mothers were unemployed. For education level, $23.9 \%$ of fathers had a college diploma or above, $45.9 \%$ of fathers reached junior college level, and $30.2 \%$ of fathers finished senior high school or below. Between the mothers, $22.0 \%$ were educated to junior high school or below, and $17.0 \%$ had a college diploma or above.

\section{Procedure}

This study was conducted in Hangzhou, a city that is in the north of Zhejiang Province on the southeast coast of China. There are 10 districts in Hangzhou City and approximately 10-15 primary schools in each district; therefore, a multistage cluster sampling design was used to select eight primary schools. First, four urban and four suburban primary schools were randomly selected. Then, three to five classes were randomly selected from Grade 3-6 in each primary school.
Inclusion criteria for students were: (1) a normal intelligence quotient, as reported by teachers; (2) no history of brain trauma or brain disease, visual or auditory dysfunction, or psychiatric disorders; (3) able to speak and read Chinese; and (4) parental consent.

This cross-sectional study was conducted between September and December 2016 by two researchers and six students with master's degrees who had experience in conducting epidemiological surveys. Before data collection, the scientific research ethics committee of Hangzhou Normal University reviewed and approved the study protocol, the informed consent forms, and the questionnaires. Permission to conduct this study in the schools was obtained from each head teacher, and informed consent was obtained from the students and their parents (through a letter sent home). When consent was given, the Children and Adolescents' Quality of Life Scale and instructions on its completion were provided to the included students, who completed the questionnaire independently. During this process, researchers were available to answer student questions. Then, questionnaires were retrieved. Each student took home the Chinese Reading Ability and its Influencing Factors questionnaire for their mother or father to complete. The students returned this questionnaire to their teacher within 1 week. Parents and students were not obliged to complete the questionnaires, even if they had provided informed consent. Anonymity and confidentiality were assured. Questionnaires with a response rate of $90 \%$ were included in the analyses. Missing data were input using medians. This study used double data entry and validation, and the logical range of each variable was considered to minimize errors. Finally, 3391 students returned the Children and Adolescents' Quality of Life Scale questionnaire with a $94.2 \%$ response rate, 3360 parents returned the Chinese Reading Ability and its Influencing Factors questionnaire with a $93.3 \%$ response rate, and 3200 families completed both questionnaires, for an $88.9 \%$ response rate.

\section{Measures}

\section{Student quality of life}

Students completed the Children and Adolescents' Quality of Life Scale that consisted of 49 items (Wu et al., 2006a, c) and measured 4 domains, and 13 dimensions. One domain evaluated social psychological function (21 items) and assessed five dimensions, including teacher-student relationship (5 items, e.g., Are you satisfied with the relationship between you and your teacher? $1=$ not at all satisfied $-4=$ very satisfied), peer relationships ( 5 items, e.g., Is your classmate friendly toward you? $1=$ not friendly $-4=$ very friendly), and parent-child relationship ( 4 items, e.g., Do you like staying with your parents?), 
learning ability and attitude (3 items, e.g., Do you remember new things easily?), and self-concept (4 items, e.g., Do you feel you are an important member of the team?), which are scored as $1=$ never $-4=$ always. Another domain evaluates physical-mental health (12 items) and assesses three dimensions, including: physical perception (5 items, e.g., Do you often feel tired after getting up?), negative emotions (4 items, e.g., Do you often feel regretful for what you have done?), and attitude toward homework (3 items, e.g., Do you need a lot of time to finish homework?), which are scored as $1=$ never $-4=$ always. A third domain evaluates living environment ( 8 items) and assesses three dimensions, including: convenience ( 2 items, e.g., Is there convenient transportation near your home? $1=$ not at all $-4=$ very convenient), opportunities for activity (3 items, e.g., Can you participate in your favorite extracurricular activities? 1 $=$ rarely $-4=$ too many opportunities), and athletic ability ( 3 items, e.g., Are you satisfied with your ability to participate in sports? $1=$ not at all $-4=$ very satisfied). Finally, there is a life satisfaction domain that assesses a self-satisfaction dimension (8 items, e.g., Are you satisfied with your sleep? $1=$ not at all satisfied $-4=$ very satisfied). Total, domain, and dimension scores were calculated, with higher scores indicating better QOL. Scores lower than two SDs below the mean were considered very poor QOL. Scores between one and two SDs below the mean were considered poor QOL. Scores that were one SD above or below the mean were considered moderate QOL. Scores that were between one and two SDs above the mean were considered better QOL. Scores that were two SDs above the mean were considered excellent (Wu et al., 2006a, c) QOL.

Total scores were transformed to a $\mathrm{T}$ score metric, which was referenced to means and SD stratified by gender, age, and region of residency for the Chinese general population. A lower $\mathrm{T}$ score represented a poorer $\mathrm{QOL}$ according to the following categorization: $\mathrm{T}<30=$ worst QOL; $30 \leq \mathrm{T}<40=$ bad QOL; $40 \leq \mathrm{T}<60=$ medium QOL; $60 \leq \mathrm{T}<70=$ good QOL; and $\mathrm{T} \geq 70=$ best QOL.

During development and validation of the Children and Adolescents' Quality of Life Scale, internal consistency and reliability for the entire questionnaire, each domain, and each dimension were evaluated as acceptable (Tavakol \& Dennick, 2011) using Cronbach's alpha (between 0.73 and 0.95). Content validity of the scale was assessed as good using a correlation coefficient between each dimension and the overall score (0.56-0.89), and the four domains and the overall score (0.52-0.83) (Wu et al., 2006b). The Children and Adolescents' Quality of Life Scale is widely used in China to assess children and adolescents aged 7-18 years. In previous studies (Peng et al., 2005a; Wu et al., 2006b), Cronbach's alpha was reported at 0.855-0.872, and content validity was reported at $0.661-0.866$. The cumulative variance contribution rate was $75.44 \%$ and the factor loading was between 0.71 and 0.89 , which indicated good construct validity. In this study, confirmatory factor analysis was used to assess the factorial structure of the Children and Adolescents' Quality of Life Scale. Findings confirmed the 13factor structure (Lance et al., 2006), because the goodness of fit index (GFI) was 0.972, the adjusted goodness of fit index (AGFI) was 0.954, the comparative fit index (CFI) was 0.948 , the Tucker-Lewis index (TLI) was 0.945 , the Chi-squared $\left(\chi^{2 /} \mathrm{df}\right)$ was 2.273 , and the root mean square error of approximation (RMSEA) was 0.045 . The cumulative percent variance was $75.44 \%$. Factor loading was used to identify whether items loaded strongly onto their hypothesized latent variable. Factor loading was from 0.48 to 0.89 , which indicated good construct validity.

\section{Student home environment}

Student home environment was defined by family SES, resource-mediated SES, reading ability, and difficulty with homework. Family SES was defined by parent education level and household income. Resource-mediated SES was defined by child and adolescent access to nutritional resources and cognitively stimulating experiences, such as reading materials and electronic devices. These variables were measured using the Chinese Reading Ability and its Influencing Factors questionnaire, which was completed by one parent from each family. Internal consistency and reliability for the entire questionnaire and each domain were evaluated using Cronbach's alpha, with values between 0.76 and 0.94 considered acceptable. Content validity of the scale was assessed as good using the correlation coefficient between each dimension and the overall score (0.67 to 0.91$)$ $(p<0.05)$. The cumulative percent variance was $72.37 \%$ and factor loading was from 0.52 to 0.87 , which indicated good construct validity.

\section{Family SES and resource-mediated SES}

In Part 1 of the Chinese Reading Ability and its Influencing Factors questionnaire, parents reported on items that assessed the general home environment, including student gender and date of birth, parent educational level, family SES (household income, annual cost of extracurricular books: $\mathrm{CNY}<300=$ 1 , CNY $300-500=2,500-800=3, \mathrm{CNY}>8000=4$ ), student dietary behavior (frequency of breakfast intake, frequency of fruit intake, and balanced diet), and the home literacy environment (student reading-related behavior, weekly use of electronic devices, and rules for use of electronic devices). Variables were defined based on previously published studies (He et al., 2014; Wang et al., 2013).

Reading-related behavior was scored based on the time spent reading each day (none $=0,0-0.5 \mathrm{~h}=1,0.5-1 \mathrm{~h}=2$, $>1 \mathrm{~h}=3$ ) plus the frequency of participation in extracurricular 
activities such as reading (2-3 times per week $=1,>3$ times per week $=2$, every day $=3$ ). Weekly use of electronic devices was scored according to the time spent watching television each day $(<1 \mathrm{~h}=0.5,1-2 \mathrm{~h}=1.5,2-3 \mathrm{~h}=2.5$, $>3 \mathrm{~h}=3$ ) multiplied by 7 days plus the frequency of computer use per week ( 1 day/week $=1$, approximately 3 days/ week $=3$, approximately 6 days per week $=6$, every day $=$ 7) multiplied by the time spent using the computer $(<1 \mathrm{~h}=$ $0.5,1-2 \mathrm{~h}=1.5,2-4 \mathrm{~h}=3,>4 \mathrm{~h}=4)$. Rules for use of electronic devices were defined according to the parental attitude concerning student television and computer use and were scored from 1 to 6 . Total scores for reading-related behavior, weekly use of electronic devices, and rules for use of electronic devices was from 0 to 27 .

\section{Student reading ability}

In Part 2 of the Chinese Reading Ability and its Influencing Factors questionnaire, parents reported on student reading ability using the Dyslexia Checklist for Chinese Children (Wu et al., 2006a, c), which consists of 57 items and 8 dimensions, including barriers to spoken language (six items, e.g., lack of competence in oral communication and not good at oral communication); problems with written expression (seven items, e.g., writing very slowly and finishing homework very late); bad reading habits (six items, e.g., reading the same sentence over again or skipping sections); attention deficit disorder (7 items, e.g., cannot concentrate during class or doing homework); visual disturbance (seven items, e.g., confuses the letters $d$ and $b$ ); disturbance in auditory perception (seven items, e.g., writing very slowly and finishing homework very late); dysgraphia (six items, e.g., does not understand normal speech, only understands when speech is slow or repeated); and difficulty understanding (nine items, e.g., often does not understand the meaning of words in sentences). The responses to each item were scored as $1=$ never $-5=$ always. Higher scores represented a lower reading ability. The eight dimensions were clustered into two categories using principal component analysis: dyslexia (defined as a specific and significant impairment in reading ability that cannot be explained by deficits in intelligence, learning opportunity, motivation, or sensory acuity (Fisher et al., 2002), and included the first six dimensions) and bad reading habits (defined as reading habits that impede reading speed or are harmful to health).

Internal consistency and reliability for the total score and the factors that composed the Dyslexia Checklist for Chinese Children were evaluated using Cronbach's alpha, with values of 0.974 and $0.752-0.901$, respectively. Factor loadings for all items were satisfactory, from 0.383 to 0.856 (Hou et al., 2018). In this study, confirmatory factor analysis was used to assess the factorial structure of the
Dyslexia Checklist for Chinese Children. Findings confirmed the eight factor structure (Lance et al., 2006), because the GFI was 0.943 , the AGFI was 0.931 , the CFI was 0.927 , the TLI was 0.925 , the $\chi^{2} / \mathrm{df}$ was 2.863 , and the RMSEA was 0.051 . The cumulative variance contribution rate was $75.68 \%$. Factor loading was used to identify whether items loaded strongly onto their hypothesized latent variable. Factor loading was between 0.41 and 0.91 , which indicated good construct validity.

\section{Student difficulty with homework}

Difficulty with homework was scored based on the need for parental pressure to ensure the homework was finished (seldom $=1$, sometime $\mathrm{s}=2$, always $=3$ ) plus the time each day required to finish the homework $(<1 \mathrm{~h}=1,1-2 \mathrm{~h}$ $=2,2-3 \mathrm{~h}=3>3 \mathrm{~h}=4$ ) according to a previously published report (He et al., 2014).

\section{Statistical Analysis}

Initially, normality, outliers, and multicollinearity were evaluated. Normality was assessed using coefficients of skewness (sk) and kurtosis (ku). Values fell within the acceptable ranges of $-0.58-1.54$ for sk and $-2.15-2.63$ for $\mathrm{ku}$. The multivariate normality test gave a value of 8.45 , which indicated that the data followed a multivariate normal distribution, because this value was $<10$. The existence of outliers were identified by Cook's distance. The maximum Cook's distance was $<0.5(0.028)$, which indicated there were no outliers in these data. Multicollinearity was tested by the tolerance rate and variance inflation factor (VIF). The findings showed no tolerance rate $<0.10$ or VIF $>10$. All the tolerance values were $>0.78$ and the VIF was $<3.9$, which indicated no multicollinearity.

Statistical analysis was performed using SPSS16.0 and AMOS22.0. A descriptive analysis was conducted using mean \pm SD for quantitative variables and frequencies \pm percentages for qualitative variables. Reliability was examined with Cronbach's alpha and content validity was measured using Pearson's correlation coefficient. Differences in QOL scores based on gender, student grade, parent education level, annual cost of extracurricular books, and student dietary habits were analyzed using a Student's t-test or analysis of variance (ANOVA) with Bonferroni post-hoc analysis. The factors that affected QOL were identified using multiple regression analysis with the four domains and total scores for QOL as dependent variables, and student gender and grade, parent education level, home literacy environment, and student reading ability as independent variables. Factors with $p<$ 0.1 in the univariate analysis were included in the multiple regression analysis. 
Based on the data from the multiple regression analysis and previously published literature, factors in the home environment, including family SES, resource-mediated SES, student reading ability, and difficulty with homework were included in structural equation modeling to analyze their effect on student QOL. The costs of extracurricular books and monthly household income were used to adjust family SES. Student reading ability, which is affected by the home literacy environment, and difficulty with homework were included in the model. Structural equation modeling was performed using AMOS24.0. A Chi-squared $\chi^{2}$ test was used to assess model fit. In addition, the CFI, TLI, RMSEA, and the standardized root mean square residual (SRMR) were used to assess goodness of fit of models. Acceptable criteria were set according to guidelines reported in literature: CFI $>0.93$, TLI $>0.90$, RMSEA $<0.08$, and SRMR $<0.08$. Full information maximum likelihood with robust standard errors (MLR) was adopted to deal with missing data and non-normality. The level of significance in the analyses was set to 0.05 .

\section{Results}

\section{Student Quality of Life}

Overall, $15.1 \%$ of students had the worst QOL, $4.3 \%$ had a bad QOL, $62.5 \%$ had a medium QOL, $14.4 \%$ had a good QOL, and 3.7\% of had the best QOL. 80.6\% of students had a medium or better QOL.

Between the four domains assessed by the QOL measure, scores for psychosocial function, physical-mental health, and life satisfaction were significantly higher among female students compared with male students $(p<0.001)$. Scores for psychosocial function, physical-mental health, living environment, and life satisfaction were significantly higher among younger students (Grade 3 or 4) compared with older students (Grade 5 or 6$)(p<0.001)$. Scores for psychosocial function and living environment were significantly higher among students residing in urban regions compared to students residing in suburban regions $(p<$ 0.01 ), and scores for life satisfaction was significantly higher among students residing in suburban regions $(p<$ 0.01) (Table S1).

\section{Influence of Parents Educational Level and Family SES on Student Quality of Life}

Scores for all four domains assessed by the QOL measure (psychosocial function, physical-mental health, living environment, life satisfaction) significantly increased with increases in parent educational level and family SES $(p<$ 0.05) (Table S2).

\section{Influence of Student Eating Habits on their Quality of Life}

Scores for psychosocial function, physical-mental health, and life satisfaction were significantly increased in students who ate breakfast every day compared with those who ate breakfast less frequently $(p<0.05)$. Scores for psychosocial function, physical-mental health, living environment, and life satisfaction were significantly increased in students who ate fruit everyday compared with those who ate fruit often or seldom and in those who ate fruit often compared with those who ate fruit seldom $(p<0.05)$. Scores for psychosocial function, physical-mental health, living environment, and life satisfaction were significantly increased in students who ate a balanced diet compared with those who ate predominantly meat or vegetables, and scores for psychosocial function, physical-mental health, and living environment were significantly increased in students who ate predominantly meat compared with predominantly vegetables $(p<0.05)$ (Table S3).

\section{Multiple Linear Regression Analysis}

Univariate analysis and multiple linear regression analysis were performed using the four domains assessed by the QOL measure (psychosocial function, physical-mental health, living environment, life satisfaction) as dependent variables and student gender, age, region of residence, family SES (parent education level, household income), dietary behavior (breakfast intake, fruit intake, balanced diet), and home literacy environment (reading-related behavior, weekly use of electronic devices, rules for use of electronic devices at home), as independent variables. Factors with $p<0.1$ on univariate analysis were included in multiple regression analysis. The findings revealed eight factors were associated with higher psychosocial function, including young age (junior grades), having a father with a college level or above of education, household income CNY 10,000-15,000, frequent breakfast consumption, daily intake of fruit, good reading-related behavior, and limited time spent using electronic devices; the most influential factor was reading-related behavior. Eight factors were associated with higher physical-mental health, including young age (Grade 3 or 4), household income CNY 10,000-15,000, frequent breakfast consumption, balanced diet, daily intake of fruit, good reading-related behavior, limited time spent using electronic devices, and lenient rules for use of electronic devices. The most influential factor was reading-related behavior. Eight factors were associated with a better living environment, including living in an urban area, having a father with college level or above of education, having a mother with a junior college level of education, frequent breakfast consumption, daily intake of fruit, 
Table 1 Multivariate linear regression for students quality of life scores (standardized coefficients)

\begin{tabular}{|c|c|c|c|c|c|c|}
\hline \multirow[t]{2}{*}{ Independent variables } & \multirow[t]{2}{*}{ Categories } & \multicolumn{5}{|l|}{ Quality of life } \\
\hline & & $\begin{array}{l}\text { Psychological } \\
\text { function }\end{array}$ & $\begin{array}{l}\text { Physical } \\
\text { mental health }\end{array}$ & $\begin{array}{l}\text { Living } \\
\text { environment }\end{array}$ & $\begin{array}{l}\text { Life } \\
\text { satisfaction }\end{array}$ & $\begin{array}{l}\text { Total } \\
\text { score of QOL }\end{array}$ \\
\hline Residence (suburb =0) & Urban & 0.021 & -0.027 & $0.118^{*}$ & $-0.057 * *$ & 0.017 \\
\hline Grade (three, four $=0$ ) & Five and six & $-0.097 * *$ & -0.103 & -0.018 & -0.136 & $-0.11 *$ \\
\hline Gender (boys $=0$ ) & Girls & $0.086^{* *}$ & 0.022 & -0.012 & 0.028 & $0.056 * *$ \\
\hline $\begin{array}{l}\text { Students reading } \\
\text { behavior }\end{array}$ & & $0.23 *$ & $0.16^{*}$ & $0.19 *$ & $0.18^{*}$ & $0.24 *$ \\
\hline $\begin{array}{l}\text { Students time using } \\
\text { electronic devices }\end{array}$ & & $-0.062^{* *}$ & $-0.078 * *$ & -0.011 & $-0.065^{* *}$ & $-0.069 * *$ \\
\hline $\begin{array}{l}\text { Rules for using } \\
\text { electronic devices } \\
\text { at home }\end{array}$ & & 0.013 & $-0.051^{* *}$ & $0.054^{* *}$ & $-0.039 * *$ & 0.003 \\
\hline $\begin{array}{l}\text { Students } \\
\text { breakfast intake }\end{array}$ & & $0.092 * *$ & $0.084 * *$ & $0.052 * *$ & $0.087 * *$ & $0.095^{* *}$ \\
\hline \multicolumn{7}{|c|}{ Monthly household income $(<5000=0)$} \\
\hline & 5001-1000 yuan & 0.014 & 0.014 & 0.009 & -0.016 & 0.012 \\
\hline & $10001-150000$ yuan & $0.073 * *$ & $0.058 * *$ & 0.032 & 0.025 & 0.070 \\
\hline & $>15000$ yuan & 0.020 & 0.008 & $0.054 * *$ & -0.006 & 0.021 \\
\hline \multicolumn{7}{|c|}{ Father's education (junior high and below $=0$ ) } \\
\hline & Senior high school & 0.019 & 0.008 & 0.022 & 0.003 & 0.022 \\
\hline & Associate degree & 0.025 & 0.021 & 0.027 & 0.011 & 0.030 \\
\hline & $\begin{array}{l}\text { Bachelor's degree } \\
\text { and above }\end{array}$ & $0.045^{* *}$ & -0.005 & $0.056^{* *}$ & 0.003 & 0.027 \\
\hline \multicolumn{7}{|c|}{ Mother's education (junior high and below $=0$ ) } \\
\hline & Senior high school & 0.015 & 0.014 & 0.021 & 0.018 & 0.023 \\
\hline & Associate degree & 0.011 & 0.006 & $0.061 * *$ & -0.002 & 0.019 \\
\hline & $\begin{array}{l}\text { Bachelor's degree } \\
\text { and above }\end{array}$ & 0.012 & 0.003 & $0.054^{* *}$ & 0.002 & 0.017 \\
\hline \multicolumn{7}{|c|}{ Students balanced diet intake (more vegetables $=0$ ) } \\
\hline & More meat & 0.020 & $-0.045^{* *}$ & $0.053 * *$ & -0.002 & 0.014 \\
\hline & Balanced diet & 0.018 & $0.046^{* *}$ & $0.072 * *$ & $0.076 * *$ & $0.064 * *$ \\
\hline \multicolumn{7}{|c|}{ Students vegetable and fruit intake (never $=0$ ) } \\
\hline & Often & 0.021 & 0.013 & 0.012 & 0.004 & 0.022 \\
\hline & Everyday & $0.068^{*}$ & $0.061 * *$ & $0.087 *$ & $0.065 * *$ & $0.089 * *$ \\
\hline
\end{tabular}

$*<0.01 ; * *<0.05$

good reading-related behavior, and strict rules for use of electronic devices. The most influential factor was readingrelated behavior. Eight factors were associated with better life satisfaction, including living in a rural area, young age (Grade 3 or 4), frequent breakfast consumption, balanced diet, daily intake of fruit, good reading-related behavior, limited time spent using electronic devices, and lenient rules for use of electronic devices. The most influential factor was reading-related behavior (Table 1).

\section{Structural Equation Modeling}

Structural equation modeling was used to describe the influence of the home environment on student QOL
(Fig. 1). Dietary behavior, difficulty with homework, reading ability, home reading environment and other influencing factors were identified using univariate analysis, multiple linear regression analysis, and exploratory factor analysis. Measurement and structural models were constructed. The measurement model was tested with confirmatory factor analysis. The strength of relationships in the structural model were estimated using correlation coefficients generated by AMOS24.0. Model fit was assessed, and the model was respecified. The final model had good fit, because $\chi^{2} / \mathrm{df}$, GFI, AGFI, NFI, IFI, and CFI were $>0.9$, and the RMSEA was $<0.05$ (Table 2).

The estimated path coefficients from one independent latent variable to the dependent latent variable are 


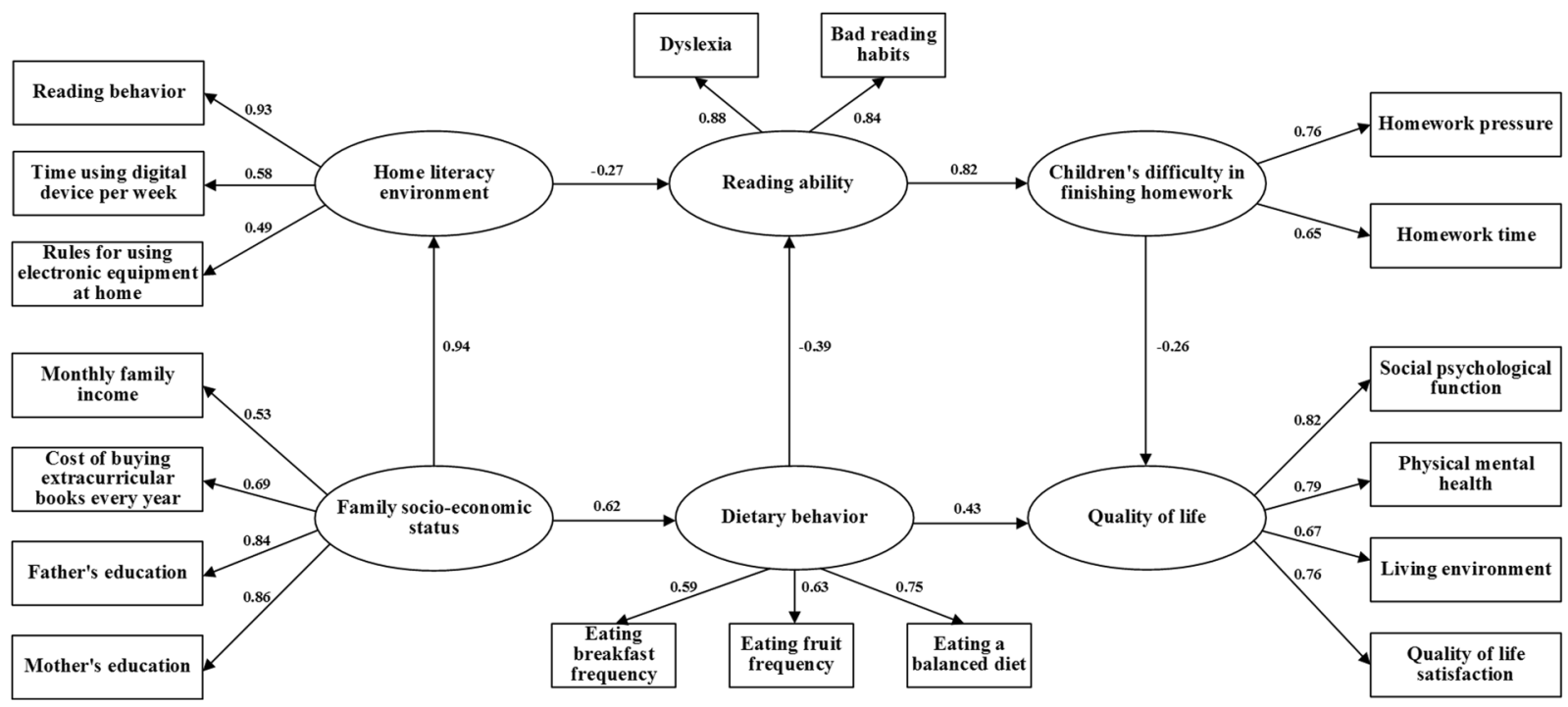

Fig. 1 Structural equation model

Table 2 Fit indices of final model

\begin{tabular}{lccccccc}
\hline Fit indices & GFI & AGFI & CFI & NFI & IFI & $\chi^{2} / \mathrm{df}$ & RMSEA \\
\hline $\begin{array}{l}\text { Reference } \\
\text { value scale }\end{array}$ & $>0.9$ & $>0.9$ & $>0.9$ & $>0.9$ & $>0.9$ & $<5$ & $<0.08$ \\
$\begin{array}{l}\text { Fitted value } \\
0.95\end{array}$ & 0.94 & 0.93 & 0.92 & 0.93 & 5.44 & 0.041 \\
\hline
\end{tabular}

$G F I$ goodness of fit index, $A G F I$ adjusted goodness of fit index, $C F I$ comparative fit index, $N F I$ normed fit index, IFI incremental fit index, $R M S E A$ root mean square error of approximation

summarized in Table 3. The direct effect of parent educational level on family SES was positive and significant. The direct effect of family SES on dietary behavior and the home literacy environment was positive and significant. The direct effect of dietary behavior on reading ability was negative and significant. The direct effect of the home literacy environment on reading ability was negative and significant. The direct effect of reading ability on difficulty with homework was positive and significant. The total effect of parent education level on family SES, dietary behavior, and the home literacy environment was positive and significant. The total effect of parent education level on reading ability and difficulty with homework was negative and significant. The total effect of family SES on dietary behavior and the home literacy environment was positive and significant. The total effect of family SES on reading ability and difficulty with homework was negative and significant. The total effects of dietary behavior and the home literacy environment on reading ability and difficulty with homework were negative and significant. The total effect of reading ability on difficulty with homework was positive and significant.

The estimated path coefficients from latent variables to QOL are summarized in Table 4. The direct effect of dietary behavior on QOL was positive and significant. The direct effect of difficulty with homework on QOL was negative and significant. The total effect of family SES (parent education level, household income), dietary behavior, home literacy environment, and reading ability on QOL was positive and significant and the total effect of difficulty with homework was negative and significant.

\section{Discussion}

This study investigated the QOL in children and adolescents residing in urban and suburban areas in Hangzhou City, Zhejiang Province, China. Compared with national data (Wu et al., 2006b), the overall QOL for primary school students in Hangzhou City was good, because $80.6 \%$ of students had a medium or better QOL. Compared with children and adolescents in the general Chinese population (Wu et al., 2006a, c), children and adolescents residing in Hangzhou City had lower scores for psychosocial function (3.04 \pm 0.47 versus national $3.72 \pm 0.71)$ and living environment $(2.73 \pm 0.54$ versus national $3.67 \pm 0.79)$ and higher scores for physical-mental health $(3.04 \pm 0.49$ versus national $2.33 \pm 0.61)$ and life satisfaction $(3.11 \pm 0.44$ versus national $1.52 \pm 0.44$ ). These data suggest that strategies should be implemented to improve the psychosocial function and living environment of children and adolescents residing in Hangzhou City. However, overall QOL and several aspects of QOL (psychological function, physical mental health, living environment, and life satisfaction) were significantly higher in children and adolescents residing in Hangzhou City compared to primary school students in Hubei province (Huang \& Shan, 2006), 
Table 3 Standardized total effect, standardized direct effect, and standardized indirect effect between latent variables

\begin{tabular}{|c|c|c|c|c|}
\hline Independent variable & Dependent variable & $\begin{array}{l}\text { Standardized } \\
\text { total effect }\end{array}$ & $\begin{array}{l}\text { Standardized } \\
\text { direct effect }\end{array}$ & $\begin{array}{l}\text { Standardized } \\
\text { indirect effect }\end{array}$ \\
\hline \multirow[t]{4}{*}{ Family socioeconomic status } & Students dietary behavior & $0.62 *$ & $0.62 *$ & 0.00 \\
\hline & Home literacy environment & $0.94 *$ & $0.94 *$ & 0.00 \\
\hline & Students reading ability & $-0.49 *$ & 0.00 & $-0.49 *$ \\
\hline & $\begin{array}{l}\text { Students difficulty in finishing } \\
\text { homework }\end{array}$ & $-0.41 * *$ & 0.00 & $-0.41 * *$ \\
\hline \multirow[t]{2}{*}{ Students dietary behavior } & Students reading ability & $-0.39 * *$ & $-0.39 * *$ & 0.00 \\
\hline & $\begin{array}{l}\text { Students difficulty in finishing } \\
\text { homework }\end{array}$ & $-0.32 * *$ & 0.00 & $-0.32 * *$ \\
\hline \multirow[t]{2}{*}{ Home literacy environment } & Students reading ability & $-0.27 * *$ & $-0.27 * *$ & 0.00 \\
\hline & $\begin{array}{l}\text { Students difficulty in finishing } \\
\text { homework }\end{array}$ & $-0.22 * *$ & 0.00 & $-0.22 * *$ \\
\hline Students reading ability & $\begin{array}{l}\text { Students difficulty finishing } \\
\text { homework }\end{array}$ & $0.82 *$ & $0.82 *$ & 0.00 \\
\hline
\end{tabular}

$*<0.01 ; * *<0.05$

Table 4 Standardized total effect, standardized direct effect, and standardized indirect effect of latent variables on students quality of life

\begin{tabular}{lccc}
\hline Latent variables & Total effect & Direct effect & Indirect effect \\
\hline $\begin{array}{l}\text { Family } \\
\text { socioeconomic status }\end{array}$ & $0.362^{*}$ & 0.000 & $0.362^{*}$ \\
$\begin{array}{l}\text { Students dietary } \\
\text { behavior }\end{array}$ & $0.513^{*}$ & $0.430^{*}$ & $0.083^{* *}$ \\
$\begin{array}{l}\text { Home literacy } \\
\text { environment }\end{array}$ & $0.0575^{* *}$ & 0.000 & $0.0575^{* *}$ \\
$\begin{array}{l}\text { Students reading ability } \\
\text { Student' difficulty with }\end{array}$ & $-0.213^{* *}$ & 0.000 & $-0.213^{* *}$ \\
homework & & $-0.260^{* *}$ & 0.000 \\
\hline
\end{tabular}

$* *<0.05, *<0.01$

Shanghai, and Suzhou (Peng et al., 2005b; Shen et al., 2004). This might be explained by the geographical location of the children and adolescents. Previous studies showed that QOL scores for primary school students varied between regions and were higher in cities than in rural areas (Chen et al., 2007). Accordingly, evidence suggests (Fang, 2001) that student QOL is a comprehensive indicator reflecting health and living standards, varying with socio-economic and cultural levels.

Previous studies that investigated similar topics always used traditional statistical methodologies (C et al., 2014; Paula et al., 2012; Wang et al., 2007), which did not evaluate the effect of latent variables. In addition, variables might be collinear by chance, which might lead to the incorrect identification of relevant predictors in the statistical model. In this study, by using structural equation modeling, the direct (parent education level and monthly household income) and indirect effects of family SES on student QOL by mediating variables, such as dietary behavior and the home literacy environment were analyzed. Bradley et al, (Bradley \& Corwyn, 2002) reported that accessibility to resources and cultural activities was a mediating variable between SES and child intelligence and behavior. The home literacy environment is an important mediating variable. A good home literacy environment provides resources for the development of a child's reading ability, and it is a protective factor against dyslexia (He et al., 2014; Noble et al., 2006; Sun et al., 2013). Since structural equation modeling investigates indirect effects, reading ability and difficulty with homework were included as factors in the home environment and were analyzed as mediating variables in this study.

Evidence suggests that fruit and vegetable intake and the variety of fruit consumed are associated with some aspects of QOL in children (Matthews et al., 2014), and an inappropriate diet and lack of breakfast might be important environmental risk factors that lead to learning disorders in children (Wang et al., 2013). In addition, it has been reported that parent beliefs and behaviors (Davis-Kean 2005; He et al., 2014) were indirectly related to child academic achievement. Therefore, in this study, student eating habits were chosen as a measure of nutritional resources, and the home literacy environment was chosen as a measure of student exposure to cognitively stimulating experiences. The results showed that dietary behaviors and the home literacy environment of students living in Hangzhou City directly affected their QOL by influencing their reading ability and their ability to do homework. Family SES indirectly affected student QOL. These data imply that a higher family SES, as shown by well-educated parents and a high household income, healthy dietary behaviors among children and adolescents, and a good home literacy environment, could improve student reading ability, decrease risks of dyslexia, 
improve student ability to do homework, and enhance student QOL. Similar to the results of this study, previous research showed that SES was not related to child mental health or psychological well-being in a sample of 19,487 school-aged children collected from the 2013-2014 China Education Panel Survey; however, SES indirectly affected child mental health and psychological well-being through parent-child relations, peer relations, and teacher-student relations (Jarman et al., 2015; Ge, 2017).

Previous studies have shown that there is an association between parental education level and nutrition in children. In Europe, the IDEFICS (identification and prevention of dietary- and lifestyle-induced health effects in children and infants) cohort study (Arvidsson et al., 2017) of 16,229 children aged 2-9 years revealed that children of parents with a lower level of education had a higher sugar and fat intake than children of parents with a higher level of education. Studies of 34,366 children in Brazil and 7474 children in the United Kingdom found that the unhealthy dietary habits of children were associated with a low level of maternal education (Cribb et al., 2011; Saldiva et al., 2014). In the United States (Shonkoff et al., 2017) a study of 599 parent-child dyads showed that clearly explained parent rules about the types of foods children could eat might decrease sugar intake.

A good home literacy environment provides children and adolescents direct and indirect learning opportunities and encourages a culture of continuous learning. Children from low-SES families often lack access to cognitively stimulating resources, such as educational materials and outdoor activities that enhance cognitive ability. Previous reports (Noble et al., 2006; Park et al., 2017) show that family SES, time parents spend reading with children, and the number of books in a home are associated with the home literacy environment; student weekday and weekend screen time decrease as parental education level increases (Sharif \& Sargent, 2006); and a good home literacy environment might improve student reading abilities (He et al., 2014; Noble et al., 2006; Sun et al., 2013).

The precise determination of the processes how the home environment influences QOL in children and adolescents is challenging; therefore, this study has several limitations. First, it was a cross-sectional study and causality could not be inferred. Second, factors that contribute to QOL are complex and important indicators might not have been considered in this model, including student to parental exposure to stress inducing conditions and health-relevant behaviors or lifestyle.

In conclusion, this study showed that the home environment influenced the QOL in children and adolescents in Hangzhou City, China. These findings emphasize that children and adolescents should have access to good nutrition and cognitively stimulating experiences to enhance their well-being and provide them with social and academic advantages.

Acknowledgements The project was supported by Provincial Natural Science Foundation of Zhejiang (No. LQ14H260001).

\section{Compliance with Ethical Standards}

Conflict of Interest The authors declare no competing interests.

Publisher's note Springer Nature remains neutral with regard to jurisdictional claims in published maps and institutional affiliations.

Open Access This article is licensed under a Creative Commons Attribution 4.0 International License, which permits use, sharing, adaptation, distribution and reproduction in any medium or format, as long as you give appropriate credit to the original author(s) and the source, provide a link to the Creative Commons license, and indicate if changes were made. The images or other third party material in this article are included in the article's Creative Commons license, unless indicated otherwise in a credit line to the material. If material is not included in the article's Creative Commons license and your intended use is not permitted by statutory regulation or exceeds the permitted use, you will need to obtain permission directly from the copyright holder. To view a copy of this license, visit http://creativecommons. org/licenses/by/4.0/.

\section{References}

Arvidsson, L., Eiben, G., Hunsberger, M., De Bourdeaudhuij, I., Molnar, D., \& Jilani, H. (2017). Bidirectional associations between psychosocial well-being and adherence to healthy dietary guidelines in European children: prospective findings from the IDEFICS study. BMC Public Health, 17(1), 926. https://doi. org/10.1186/s12889-017-4920-5

Balazs, J., Miklosi, M., Toro, K. T., \& Nagy-Varga, D. (2016). Reading disability and quality of life based on both self- and parent-reports: importance of gender differences. Frontiers in Psychology, 7, 1942

Boe, T., Serlachius, A. S., Sivertsen, B., Petrie, K. J., \& Hysing, M. (2018). Cumulative effects of negative life events and family stress on children's mental health: the Bergen Child Study. Social Psychiatry and Psychiatric Epidemiology, 53(1), 1-9. https://doi. org/10.1007/s00127-017-1451-4

Bradley, R., \& Corwyn, R. (2002). Socioeconomic status and child development. Annual Review of Psychology, 53, 371-399

Chen, L., Wu, H., \& Mai, J. (2007). Comparison of quality of life among primary and secondary school students between urban and rural in Beijing and Guangzhou. Chinese Journal of Social Medicine, 24(4), 268-270

Cribb, V. L., Jones, L. R., Rogers, I. S., Ness, A. R., \& Emmett, P. M. (2011). Is maternal education level associated with diet in 10year-old children? Public Health Nutrition, 14(11), 2037-2048. https://doi.org/10.1017/s136898001100036x

Daniel, T. L. S., \& Britta, M. L. (2007). A comprehensive review of quality of life (QOL) research in Hong Kong. Scientific World Journal, 7, 1222-1229

Davis-Kean, P. E. (2005). The influence of parent education and family income on child achievement: the indirect role of parental expectations and the home environment. Journal of Family Psychology, 19(2), 294-304. https://doi.org/10.1037/0893-3200.19.2.294

Fang, J. (2001). Determination of quality of life and its application, Vol. 1. Beijing: Beijing Medical University Press 
Fisher, S. E., Francks, C., Marlow, A. J., MacPhie, I. L., Newbury, D. F., \& Cardon, L. R. (2002). Independent genome-wide scans identify a chromosome 18 quantitative-trait locus influencing dyslexia. Nature Genetics, 30(1), 86-91. https://doi.org/10.1038/ng792

Ge, T. (2017). Effect of socioeconomic status on children's psychological well-being in China: the mediating role of family social capital. Journal of Health Psychology, 1359105317750462. https://doi.org/10.1177/1359105317750462

He, Z., Shao, S., Zhou, J., Ke, J., Kong, R., \& Guo, S. (2014). Does long time spending on the electronic devices affect the reading abilities? A cross-sectional study among Chinese school-aged children. Research in Developmental Disabilities, 35(12), 3645-3654. https://doi.org/10.1016/j.ridd.2014.08.037

Hong, R. (2007). Effect of economic inequality on chronic childhood undernutrition in Ghana. Public Health Nutrition, 10(4), 371-378. https://doi.org/10.1017/s1368980007226035

Hosokawa, R., \& Katsura, T. (2017). A longitudinal study of socioeconomic status, family processes, and child adjustment from preschool until early elementary school: the role of social competence. Child Adolescent Psychiatry Mental Health, 11, 62 https://doi.org/10.1186/s13034-017-0206-Z

Hou, F., Qi, L., Liu, L., Luo, X., Gu, H., \& Xie, X. (2018). Validity and reliability of the dyslexia checklist for Chinese children. Frontiers in Psychology, 9, 1915 https://doi.org/10.3389/fpsyg. 2018.01915

Huang, Y., Han-Rong, W. U., \& Shan, L. U. (2006). Comparison of quality of life between city and countryside among primary and secondary school students in Hubei. Chinese Journal of School Health, 27(1), 34-35.

Jarman, M., Ogden, J., Inskip, H., Lawrence, W., Baird, J., \& Cooper, C. (2015). How do mothers manage their preschool children's eating habits and does this change as children grow older? A longitudinal analysis. Appetite, 95, 466-474. https://doi.org/10. 1016/j.appet.2015.08.008

Jin, H. H., \& Lu, Y. (2017). Academic performance of Texas public schools and its relationship with students' physical fitness and socioeconomic status. International Journal of Applied Geospatial Research, 8(3), 37-52

Joreskog, K. G., \& Sorbom, D. (1979). Advances in Factor Analysis and Structural Equation Models. Cambridge, MA: Abt Books

Lance, C. E., Marcus, M. B., \& Michels, L. C. (2006). The sources of four commonly reported cutoff criteria: what did they really say? Organ Research Methods, 9, 202-220.

Lee, D., \& Jackson, M. (2017). The simultaneous effects of socioeconomic disadvantage and child health on children's cognitive development. Demography, 54(5), 1845-1871. https://doi.org/10. 1007/s13524-017-0605-z

Maatta, S., Konttinen, H., Haukkala, A., Erkkola, M., \& Roos, E. (2017). Preschool children's context-specific sedentary behaviours and parental socioeconomic status in Finland: a crosssectional study. BMJ Open, 7(11), e016690 https://doi.org/10. 1136/bmjopen-2017-016690

Matthews, C., Milte, C. M., Ball, K., \& McNaughton, S. A. (2014). Associations between fruit and vegetable intake and quality of life. Journal of Nutrition \& Intermediary Metabolism, 1, 18

Meuleners, L. B., Lee, A. H., Binns, C. W., \& Lower, A. (2003). Quality of life for adolescents: assessing measurement properties using structural equation modelling. Quality of life Research, 12 (3), 283-290

Nathan, K. (2006). Reading difficulties and psychosocial problems: does social information processing moderate the link? Australian Journal of Psychology, 58(4), 171

Noble, K. G., Farah, M. J., \& McCandliss, B. D. (2006). Socioeconomic background modulates cognition-achievement relationships in reading. Cognitive Development, 21(3), 349-368. https://doi.org/10.1016/j.cogdev.2006.01.007
Park, S., Stone, S. I., \& Holloway, S. D. (2017). School-based parental involvement as a predictor of achievement and school learning environment: an elementary school-level analysis. Children \& Youth Services Review, 82, 195-206

Paula, J. S., Leite, I. C., Almeida, A. B., Ambrosano, G. M., Pereira, A. C., \& Mialhe, F. L. (2012). The influence of oral health conditions, socioeconomic status and home environment factors on schoolchildren's self-perception of quality of life. Health Quality Life Outcomes, 10, 6 https://doi.org/10.1186/14777525-10-6

Peng, N., Wang, L., \& Wang, L. (2005a). A study of life among primary and secondary school students in Shanghai. Chinese Journal of School Health, 26(4), 265-268

Peng, N., Wang, L., \& Wang, L. (2005b). A study on quality of life among primary and secondary school students in Shanghai. Chinese Journal of School Health, 26(4), 265-268

Poulain, T., Vogel, M., \& Kiess, W. (2020). Review on the role of socioeconomic status in child health and development. Current Opinion in pediatrics, 32(2), 308-314

Ran, M., Peng, L., Liu, Q., Pender, M., He, F., \& Wang, H. (2018). The association between quality of life (QOL) and health literacy among junior middle school students: a cross-sectional study. BMC Public Health, 18(1), 1183

Rashid, V., Engberink, M. F., van Eijsden, M., Nicolaou, M., Dekker, L. H., \& Verhoeff, A. P. (2018). Ethnicity and socioeconomic status are related to dietary patterns at age 5 in the Amsterdam born children and their development (ABCD) cohort. BMC Public Health, 18(1), 115 https://doi.org/10.1186/ s12889-017-5014-0

Roubinov, D. S., \& Boyce, W. T. (2017). Parenting and SES: relative values or enduring principles? Current Opinion in Psychology, $15,162-167$

Rowland, A. S., Skipper, B. J., Rabiner, D. L., Qeadan, F., Campbell, R. A., \& Naftel, A. J. (2018). Attention-deficit/hyperactivity disorder (ADHD): interaction between socioeconomic status and parental history of ADHD determines prevalence. Journal Child Psychology Psychiatry, 59(3), 213-222. https://doi.org/10.1111/ jcpp. 12775

Russell, A. E., Ford, T., \& Russell, G. (2018). The relationship between financial difficulty and childhood symptoms of attention deficit/hyperactivity disorder: a UK longitudinal cohort study. Social Psychiatry and Psychiatric Epidemiology, 53(1), 33-44. https://doi.org/10.1007/s00127-017-1453-2

Saldiva, S.R., Venancio, S.I., de Santana, A.C., da Silva Castro, A.L., Escuder, M.M., \& Giugliani, E.R. (2014). The consumption of unhealthy foods by Brazilian children is influenced by their mother's educational level. Nutrition Journal, 13(33). https://doi. org/10.1186/1475-2891-13-33

Sharif, I., \& Sargent, J. D. (2006). Association between television, movie, and video game exposure and school performance. Pediatrics, 118(4), e1061-1070. https://doi.org/10.1542/peds. 2005-2854

Shen, H., Li, H., \& Xu, Y. (2004). Survey on quality of life in Suzhou. Chinese School Medicine, 18(6), 497-498

Shonkoff, E. T., Dunton, G. F., Chou, C. P., Leventhal, A. M., Bluthenthal, R., \& Pentz, M. A. (2017). Direct and indirect effects of parent stress on child obesity risk and added sugar intake in a sample of Southern California adolescents. Public Health Nutrition, 20(18), 3285-3294. https://doi.org/10.1017/ s136898001700252x

Sohr-Preston, S. L., Scaramella, L. V., Martin, M. J., Neppl, T. K., Ontai, L., \& Conger, R. (2013). Parental socioeconomic status, communication, and children's vocabulary development: a third-generation test of the family investment model. Child Development, 84(3), 1046-1062. https://doi.org/10.1111/cdev. 12023 
Sun, Z., Zou, L., Zhang, J., Mo, S., Shao, S., \& Zhong, R. (2013). Prevalence and associated risk factors of dyslexic children in a middle-sized city of China: a cross-sectional study. PLoS One, 8 (2), e56688. https://doi.org/10.1371/journal.pone.0056688

Tavakol, M., \& Dennick, R. (2011). Making sense of Cronbach's alpha. International Journal of Medical Education, 2, 53-55. https://doi.org/10.5116/ijme.4dfb.8dfd

Wang, C., Wu, J., \& Zhao, W. (2007). Health-related quality of life among school-aged children and its association with family environment. Chinese Journal of School Health, 28(5), 423-425

Wang, Q., Wang, X., \& Peng, J. (2013). Relationship of learning disabilities with both dietary patterns and dietary behaviors in children. Chinese Journal of Child Health Care, 21(2), 141-143
Wu, H., Liu, P., \& Meng, H. (2006a). Norm, reliability and validity of children and adolescents' QOL Scale. Chinese Journal of School Health, 27(1), 18-21.

Wu, H., Li, P., \& Meng, H. (2006b). Norm, reliability and validity of children and adolescents' QOL Scale. Chinese Journal of School Health, 27(1), 10-21

Wu, H. R., Song, R. R., \& Yao, B. (2006c). The establishment of dyslexia checklist for Chinese children. Chinese Journal of School Health, 27(3), 189-190

Zou, R., Niu, G., Chen, W., Fan, C., Tian, Y., \& Sun, X. (2018). Socioeconomic inequality and life satisfaction in late childhood and adolescence: a moderated mediation model. Social Indicators Research, 136(9), 1-14 